Check for updates

Cite this: RSC Adv., 2019, 9, 5445

Received 21st December 2018 Accepted 5th February 2019

DOI: $10.1039 / c 8 r a 10478 f$

rsc.li/rsc-advances

\section{Free-standing polypyrrole/polyaniline composite film fabricated by interfacial polymerization at the vapor/liquid interface for enhanced hexavalent chromium adsorption}

\begin{abstract}
Vu Dinh Thao, ${ }^{a}$ Bach Long Giang ${ }^{b}$ and Tran Viet Thu (D) *ca
Interfacial polymerization is an innovative technique for the fabrication of polymeric films. However, the majority of studies on interfacial polymerization has focused on liquid/liquid interfaces, and little work has been done on vapor/liquid interfaces. In this paper, we present the fabrication of free-standing polypyrrole/polyaniline (PPy/PANI) composite films by interfacial polymerization at a vapor/liquid interface using $\mathrm{FeCl}_{3}$ as an oxidant. The obtained PPy/PANI composite films were characterized using Fourier transform infrared spectroscopy, X-ray diffractometry, and scanning electron microscopy. It was found that the PPy/PANI composite films consist of PANI particles evenly distributed on porous PPy film. The influence of $\mathrm{FeCl}_{3}$ concentration on the morphology of the resulting composite films was investigated. The PPy/PANI composite films show an excellent $\mathrm{Cr}(\mathrm{vl})$ adsorption capacity of $256.41 \mathrm{mg}$ $\mathrm{g}^{-1}$, much higher than that of PPy-based absorbents prepared from chemical and electrochemical polymerization routes. This work thus suggests a new route for the fabrication of PPy/PANI films with highly enhanced $\mathrm{Cr}(\mathrm{VI})$ adsorption capacity for practical applications.
\end{abstract}

\section{Introduction}

Contamination of aquatic media by heavy metals has become a serious environmental problem due to the rapid expansion of global industrial activities. ${ }^{1}$ Among all heavy metals, chromium (Cr) is extensively used in the leather tanning, electroplating, metal finishing and steel manufacturing industries, and is considered as a high priority environmental pollutant. ${ }^{2}$ Cr exists in the aqueous environment both as trivalent $(\mathrm{Cr}(\mathrm{III}))$ and hexavalent $(\mathrm{Cr}(\mathrm{vI}))$ forms. $\mathrm{Cr}(\mathrm{vI})$ is highly toxic, carcinogenic and mutagenic, and is roughly 500 times more toxic than $\mathrm{Cr}(\mathrm{III}){ }^{3,4} \mathrm{Cr}(\mathrm{VI})$ contamination causes severe human health problems such as nausea, diarrhea, liver, kidney damage and lung cancer. ${ }^{5}$ The World Health Organization has adopted an upper limit for total $\mathrm{Cr}$ content as $50 \mu \mathrm{g} \mathrm{\textrm {L } ^ { - 1 }}$ for drinking water. ${ }^{6}$

The conventional methods for $\mathrm{Cr}(\mathrm{vI})$ removal from the environment include chemical precipitation, ion exchange, electrodeposition, biological treatment, film filtration and adsorption. ${ }^{7}$ Among those techniques, adsorption has many advantages such as simplicity, low cost, high efficiency, and easy

${ }^{a}$ Department of Chemical Engineering, Le Quy Don Technical University, 236 Hoang Quoc Viet, Hanoi 100000, Vietnam. E-mail: thutv@mta.edu.vn; Tel: +84 964793732 ${ }^{b}$ NTT Institute of Hi-Technology, Nguyen Tat Thanh University, Ho Chi Minh City, Vietnam

'Institute of Research and Development, Duy Tan University, K7/25 Quang Trung, Da Nang 550000, Vietnam implementation. Up to now, a variety of adsorbents has been employed for $\mathrm{Cr}(\mathrm{vI})$ adsorption such as polyethyleneimine (PEI)silica nanocomposite, ${ }^{8}$ activated carbon (AC), ${ }^{9}$ mesoporous $\gamma$ $\mathrm{Fe}_{2} \mathrm{O}_{3}{ }^{10}$ polyacrylonitrile fiber, ${ }^{11}$ ion-exchange resins, ${ }^{12}$ and biosorbents. ${ }^{13}$ Nevertheless, the search for new adsorbent materials with high adsorption capacity, low-cost, easy separation and recovery is still in great demand.

Recently, the use of conducting polymers such as polyaniline (PANI) or polypyrrole (PPy) as adsorbents for environmental remediation has shown great promise. ${ }^{\mathbf{1 4 - 1 8}}$ These polymers have excellent environmental stability, good redox reversibility, low cost, easy synthesis and treatment, and plenty of amine and imine functional groups for specific adsorption. Binary hybrids/ composites made of these polymers such as PPy/PANI, ${ }^{\mathbf{1 6}} \mathrm{PANI} /$ $\mathrm{PEG}^{19}$ or $\mathrm{PAN} / \mathrm{PPy}^{20}$ are also efficient for the removal of metallic ions, and it was shown that the composites comprising of at least two different monomer units was proven to be superior to those made from individual ones. Among these, PPy/PANI composite fibers or particles exhibit enhanced $\mathrm{Cr}(\mathrm{vI})$ adsorption, but they tend to aggregate in aqueous environment ${ }^{\mathbf{1 6}}$ and their specific surface area is decreased. Another problem is the difficulty in recovering the material after adsorption, causing the secondary contamination. These problems are unavoidably encountered in the preparation of conducting polymers in liquid phases, and it is emerging to develop novel approaches for the fabrication of these polymers towards environmental applications. 
Interfacial polymerization has shown huge potential for preparing conductive polymers ${ }^{21-23}$ and their composites such as $\mathrm{PANI} / \mathrm{Au},{ }^{24} \mathrm{PANI} / \mathrm{Ag},{ }^{25} \mathrm{PPy} /$ graphene oxide. ${ }^{26}$ However, the majority of researches on interfacial polymerization has concentrated on liquid/liquid interfaces, and there are only few works have been carried out on vapor/liquid interfaces. ${ }^{27}$ In this work, free-standing PPy/PANI composite films were prepared by the interfacial polymerization of pyrrole and aniline gaseous monomer at vapor/liquid interface. Different from conventional chemical or electrochemical polymerization routes, in this method, the time-consuming procedure of re-distillation of monomers (before polymerization) is not necessary. Besides, this polymerization technique is also simple, efficient, and scalable. The influence of $\mathrm{FeCl}_{3}$ concentration on the structure and morphology of the films was investigated. The PPy/PANI composite films exhibit enhanced adsorption capability towards the toxic $\mathrm{Cr}(\mathrm{vI})$ ions as compared to those prepared from conventional polymerization methods, indicating that PPy/PANI composite films fabricated by interfacial polymerization at vapor/liquid interface is a promising absorbent material for environmental remediation.

\section{Experiments}

\subsection{Chemicals}

Pyrrole (99\%) and aniline (98\%) reagent grade (Sinopharm Chemical Reagent Co. Ltd.) were used as monomers. Iron chloride hexahydrate $\left(\mathrm{FeCl}_{3} \cdot 6 \mathrm{H}_{2} \mathrm{O}\right)$ (Tianjin East China Reagent Co., Ltd.) was used as oxidant. All chemicals were used without further purification.

\subsection{Preparation of free-standing PPy/PANI composite films}

The interfacial polymerization of pyrrole and aniline was carried out by taking the two monomers in equimolar proportion. In a typical procedure, a predetermined amount of $\mathrm{FeCl}_{3} \cdot 6 \mathrm{H}_{2} \mathrm{O}$ was dissolved in $40 \mathrm{~mL}$ deionized water to form $\mathrm{FeCl}_{3}$ solution of desired concentrations $(0.075 \mathrm{M} ; 0.15 \mathrm{M}, 0.3 \mathrm{M}$ or $0.5 \mathrm{M})$. This solution was transferred to a culture disk $(9 \mathrm{~cm}$ diameter) and placed inside a vacuum desiccator (central position). Two other beakers containing $1 \mathrm{~mL}$ of pyrrole or aniline each, were placed near the $\mathrm{FeCl}_{3}$ solution. A pump was used to vacuum the desiccator for about $5 \mathrm{~min}$ to induce a pressure of $-20 \mathrm{mmHg}$, which accelerates the evaporation of monomers. Then the pump was stopped, and the desiccator was kept undisturbed. After a few hours, a black-colored, freestanding PPy/PANI composite film was clearly observed on the surface of $\mathrm{FeCl}_{3}$ solution and in contact with the air. The film was transferred onto a cellulosic filter and washed with distilled water and ethanol to remove unreacted reagents and impurities. Finally, the film was naturally dried at room temperature and peeled off from the filter. For comparison, PPy films (without PANI) was also prepared in the same conditions in the absence of aniline. The films are denoted as PPy/PANI- $x$ or PPy$x$, where $x$ is the $\mathrm{FeCl}_{3}$ concentration used for the preparation of corresponding film.

\subsection{Characterization}

As-prepared PPy and PPy/PANI composite films were subjected to characterization. Scanning electron microscopy (SEM) images were taken with a Jeol JSM-6510LV scanning electron microscope. The thickness of the films was determined from SEM crosssectional images. To avoid charging during measurements, the samples were sputtered with a layer of gold prior to imaging to improve conductivity. Fourier transform infrared (FTIR) spectra were measured in the range of $400-4000 \mathrm{~cm}^{-1}$ using a Thermo Scientific Nicolet iS10 FT-IR spectrometer. Powder X-ray diffraction (XRD) patterns were measured using a Siemens D5005 diffractometer $(30 \mathrm{kV} / 40 \mathrm{~mA}, \lambda=1.5406 \AA)$. The elemental composition of PPy/PANI films was determined using energydispersive X-ray (EDX) spectroscopy, acquired on a Jeol JSM6610 LA scanning electron microscope, operated under an acceleration voltage of $15 \mathrm{kV}$ and a probe current of $1 \mathrm{nA}$.

\subsection{Batch adsorption studies}

The effect of $\mathrm{pH}$ on $\mathrm{Cr}(\mathrm{vI})$ adsorption was studied in batch adsorption experiments using $10 \mathrm{mg}$ of PPy/PANI film and $10 \mathrm{~mL}$ of $\operatorname{Cr}(\mathrm{vI})$ aqueous solutions (concentration of $\operatorname{Cr}(\mathrm{vI})$ : $10 \mathrm{mg} \mathrm{L}{ }^{-1}$ ). The adsorption experiments were carried out in $50 \mathrm{~mL}$ conical flasks on a rotary shaker operated at $200 \mathrm{rpm}$ at $\sim 25{ }^{\circ} \mathrm{C}$. Different $\mathrm{pH}$ values of 4,7 and 10 were adjusted by adding $0.1 \mathrm{M} \mathrm{NaOH}$ or $0.1 \mathrm{M} \mathrm{HCl}$ solutions. For studying adsorption equilibrium isotherm, $10 \mathrm{mg}$ of PPy/PANI film was mixed with $100 \mathrm{~mL}$ of $\mathrm{Cr}(\mathrm{vI})$ solution at different concentrations (5-100 $\mathrm{mg} \mathrm{L}^{-1}$ ) and shaken for $240 \mathrm{~min}$ at $\mathrm{pH}$ 7. Upon reaching adsorption equilibrium, the PPy/PANI film was removed and the $\mathrm{Cr}(\mathrm{vI})$ concentration was analyzed by inductively coupled plasma optical emission spectroscopy (ICP-OES) (Perkin Elmer Optima 7300DV, USA). The Cr(vI) adsorption capacity of PPy/ PANI film was calculated according to the equation:

$$
Q_{\mathrm{e}}=\left(C_{\mathrm{o}}-C_{\mathrm{e}}\right) \times V / m
$$

where $C_{\mathrm{o}}$ and $C_{\mathrm{e}}$ represent the initial and equilibrium $\mathrm{Cr}$ concentration, respectively; $V$ is the volume of aqueous solution containing $\mathrm{Cr}(\mathrm{vI})$ ions, and $m$ is the weight of the PPy/PANI film used in the experiment.

\section{Results and discussion}

\subsection{Preparation of PPy/PANI film}

The preparation of free-standing PPy/PANI composite films is schematically demonstrated in Fig. 1a. Vacuum pumping creates a low pressure $(-20 \mathrm{mmHg})$ inside the desiccator, which greatly enhance the evaporation of pyrrole and aniline molecules from their liquid state. These monomers start to polymerize upon contacting with $\mathrm{FeCl}_{3}$ oxidant at the vapor/liquid interface, forming PPy/PANI composite film in a few hours. The formation of PPy/PANI composite films at vapor/liquid interface at different durations is shown in Fig. 1b. The color of the growing film changes from light purple $(1 \mathrm{~h})$ to dark purple $(2 \mathrm{~h})$, blackish purple $(3 \mathrm{~h})$, greenish black $(4 \mathrm{~h})$ and black (5 h), demonstrating its evolution in morphology, 
microstructure, and physical dimension (thickness). Furthermore, the PPy/PANI composite film entirely covers $\mathrm{FeCl}_{3}$ solution surface, indicating that the nucleation occurs at the whole vapor/ liquid interface. The as-prepared PPy/PANI composite film can be transferred to a substrate, washed to remove unbounded $\mathrm{FeCl}_{3}$, let to dry, and peeled off using tweezers. The isolated PPy/PANI composite films were moderately flexible and free-standing without any substrates (Fig. 1c), indicating its sustainability. For comparison, we also performed similar experiments using pyrrole or aniline as the only monomer. In the absence of aniline, the interfacial polymerization of pyrrole also resulted in a freestanding PPy film which exhibited a blackish green color, similar to previous report. ${ }^{27}$ However, the PPy film was much thinner and mechanically weak. On the contrary, if only aniline monomers were used, the as-formed PANI particles were dramatically dispersed into the aqueous phase to form bulky precipitates, without the formation of a film.

\subsection{IR spectra}

In order to recognize the functional groups present in polymer films, we have measured and compared IR spectra of bulky PANI, PPy film, and PPy/PANI composite film (Fig. 2). In the IR spectrum of bulky PANI (green line), the peaks at 1604 and $1496 \mathrm{~cm}^{-1}$ are assigned to the $\nu(\mathrm{C}=\mathrm{C})$ in quinoid phenyl ring and $\nu(\mathrm{C}=\mathrm{C})$ in benzenoid phenyl ring, respectively. The peak at $1297 \mathrm{~cm}^{-1}$ is attributed to $\nu(\mathrm{C}-\mathrm{N})$ of a secondary aromatic amine, and the peak at $1244 \mathrm{~cm}^{-1}$ is attributed to the $\delta(\mathrm{C}-\mathrm{H})$ in benzene plane. The peak at $1154 \mathrm{~cm}^{-1}$ is from $\nu(\mathrm{C}=\mathrm{N})$ of $\mathrm{N}=\mathrm{Q}=\mathrm{N}$ ( $\mathrm{Q}$ is quinoid ring), and the peak at $812 \mathrm{~cm}^{-1}$ is assigned to the $\delta(\mathrm{C}-\mathrm{H})$ of 1,4-substituted phenyl ring stretch. These peaks are similar to those of PANI synthesized by a conventional method. ${ }^{28}$ The IR spectrum of PPy film (red line) shows characteristic peaks/bands of PPy at $1509 \mathrm{~cm}^{-1}$ (pyrrole ring stretching), $1426 \mathrm{~cm}^{-1}$ (conjugated $\mathrm{C}-\mathrm{N}$ stretching), $1266 \mathrm{~cm}^{-1}$ (=C-H in-plane deformation), $1107 \mathrm{~cm}^{-1}(\mathrm{C}-\mathrm{H}$ stretching), $1082 \mathrm{~cm}^{-1}$ (C-H in-plane bending), $1000 \mathrm{~cm}^{-1}(\mathrm{~N}-$ $\mathrm{H}$ in-plane deformation), $960 \mathrm{~cm}^{-1}$ (C-C out-of-plane), $811 \mathrm{~cm}^{-1}$ (-C-H out-of-plane), and $692 \mathrm{~cm}^{-1}$ (C-C out-of-

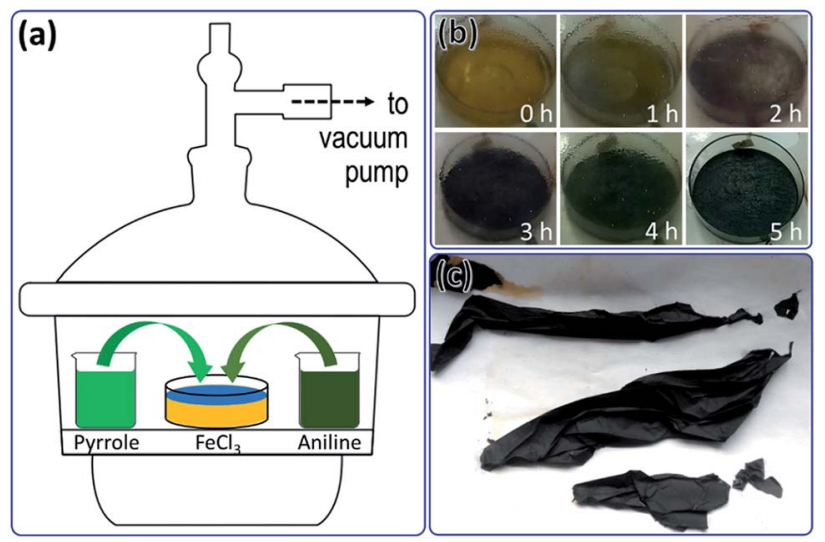

Fig. 1 (a) Schematic preparation of PPy/PANI composite films. (b) Digital photos of PPy/PANI composite films taken after $0,1,2,3,4$, and 5 h. (c) Free-standing PPy/PANI composite films.

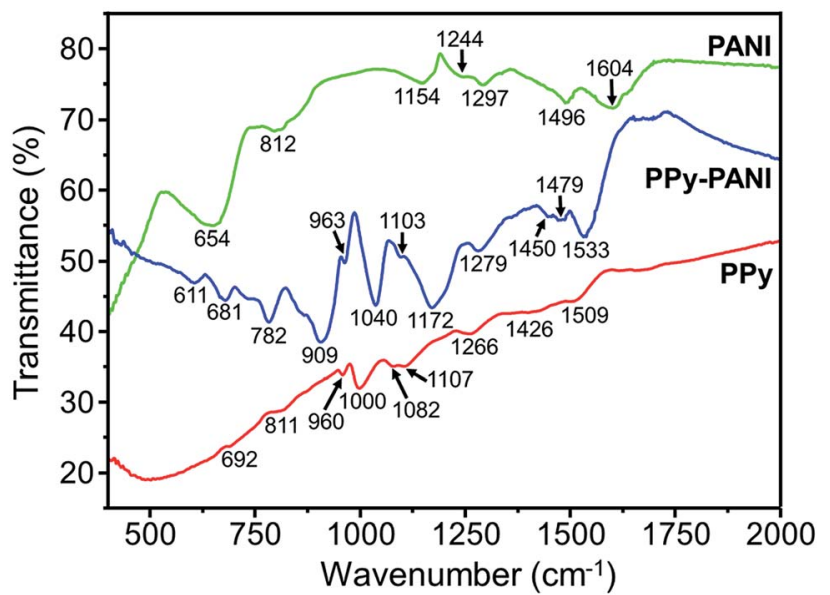

Fig. 2 FTIR spectra of bulky PANI (green), PPy film (red) and PPy/PANI films (blue).

plane ring deformation). ${ }^{29}$ In the IR spectrum of PPy/PANI composite film (blue line), the peak at $1533 \mathrm{~cm}^{-1}$ can be assigned to $\mathrm{C}-\mathrm{C}$ stretching vibration in the pyrrole ring and quinone stretching vibration in the phenyl ring. ${ }^{30}$ The peaks at 1479 and $1450 \mathrm{~cm}^{-1}$ can be attributed to conjugated $\mathrm{C}-\mathrm{N}$ stretching vibration in the pyrrole ring and benzene in the phenyl ring. ${ }^{31,32}$ The peak at $1279 \mathrm{~cm}^{-1}$ can be attributed to $\nu(\mathrm{C}-$ $\mathrm{N}$ ) of a secondary aromatic amine. The strong peak at $1172 \mathrm{~cm}^{-1}$ is assigned to $\nu(\mathrm{C}=\mathrm{N}$ ) of $\mathrm{N}=\mathrm{Q}=\mathrm{N}$ ( $\mathrm{Q}$ is quinoid ring). The peak at $1103 \mathrm{~cm}^{-1}$ can be assigned to $\mathrm{C}-\mathrm{H}$ in-plane bending on 1,2,4-substituted benzene of PANI. ${ }^{33}$ The 1,2 , or 4 positions on the benzene ring are likely favorable sites for the pyrrole units to attach on. The peaks at 1040 and $963 \mathrm{~cm}^{-1}$ can be attributed to $\mathrm{C}-\mathrm{H} / \mathrm{N}-\mathrm{H}$ in-plane and $\mathrm{C}-\mathrm{C}$ out-of-plane ring deformation vibrations, correspondingly. ${ }^{30}$ The peak at $909 \mathrm{~cm}^{-1}$ is attributed to $\mathrm{C}-\mathrm{H}$ deformation. The peak at $782 \mathrm{~cm}^{-1}$ is possibly due to the $\delta(\mathrm{C}-\mathrm{H})$ of 1,4 -substituted phenyl ring stretch, and the peak at $681 \mathrm{~cm}^{-1}$ can be given by $\mathrm{C}-\mathrm{C}$ out of plane ring deformation. The peak at $611 \mathrm{~cm}^{-1}$ can be assigned to $\pi-\pi *$ transition of the benzenoid ring. ${ }^{32,34}$ All the main characteristic peaks of PANI and PPy were observed in PPy/PANI composite films. However, the position of several peaks slightly deviates from neat PANI and PPy. For example, the peak presenting stretching vibrations of the pyrrole rings in PPy/PANI is shifted to a higher frequency region $\left(1533 \mathrm{~cm}^{-1}\right)$, as compared to that of the neat PPy $\left(1509 \mathrm{~cm}^{-1}\right)$. This shift might be associated with the changes of electronic properties as a result of $\pi-\pi$ interactions between PPy and PANI moieties in polymer backbone. ${ }^{35}$ It has been previously observed that this peak shift depends on a number of factors such as PPy : PANI molar ratio. Our FTIR analysis confirms the presence of both PPy and PANI moieties in the synthesized PPy/PANI composite films.

\subsection{XRD}

Conducting polymers are semi-crystalline materials in which the crystallinity is associated with intra- and inter-chain interactions. $^{36}$ These interactions induce the local alignment of polymer chains and formation of single/multiple helices or 
chain folding, forming ordered nanocrystallines which are bounded by amorphous domains. As shown in Fig. 3, PPy/PANI composite films prepared with different $\mathrm{FeCl}_{3}$ concentrations exhibit very similar XRD patterns, suggesting that those films have comparable aggregated structures and polymer backbones. PPy/PANI composite films exhibit two characteristic peaks at $2 \theta$ of $c a .24 .4^{\circ}$ and $26.8^{\circ}$. When $\mathrm{FeCl}_{3}$ concentration increases from 0.075 to $0.5 \mathrm{M}$, the intensity of the first peak increases while that of the second peak decreases, suggesting minor changes in the structure of the films. These two peaks are actually not clearly distinguished (especially at $\mathrm{FeCl}_{3}$ concentration of $0.15 \mathrm{M}$ ) but merged and overlapped onto each other's, forming a broad and asymmetric peak, centered at $25.6^{\circ}$. This peak corresponds to an approximate inter-backbone packing distance ( $d$-spacing) of $3.5 \AA$ as calculated using Bragg's law. This value is smaller than that of free-standing PPy films prepared by chemical interfacial polymerization ${ }^{37}$ or PPy microtubules prepared by conventional chemical oxidation ${ }^{38}$ ( $c a$. $3.7 \AA$ @). Meanwhile, the relative intensity of this peak diminishes when $\mathrm{FeCl}_{3}$ concentration increases, indicated declined crystallinity. The fact that PPy/PANI films synthesized at low concentrations of $\mathrm{FeCl}_{3}$ exhibited higher order can be attributed to lower rate of film growth and strengthened macroscopic arrangement of PPy/ PANI chains. The peak positions of all PPy/PANI films prepared at different $\mathrm{FeCl}_{3}$ concentrations were nearly the same, indicating that the domain length of the PPy/PANI polymer chains is independent on $\mathrm{FeCl}_{3}$ concentration.

\subsection{SEM observation}

The morphology of PPy films prepared without PANI was studied in order to understand how PPy grows into a film. Fig. 4 shows SEM images of PPy films prepared for $1 \mathrm{~h}$ at different $\mathrm{FeCl}_{3}$ concentrations. At a $\mathrm{FeCl}_{3}$ concentration of $0.075 \mathrm{M}$, the film displays a high density of pore-like features with irregular shape and various diameters. Upon increasing $\mathrm{FeCl}_{3}$ concentration to $0.15 \mathrm{M}$, the density of these features increases and their average size decreases. It can be attributed to the increased number of $\mathrm{FeCl}_{3}$ oxidant sites on vapor/liquid interface, which increases the

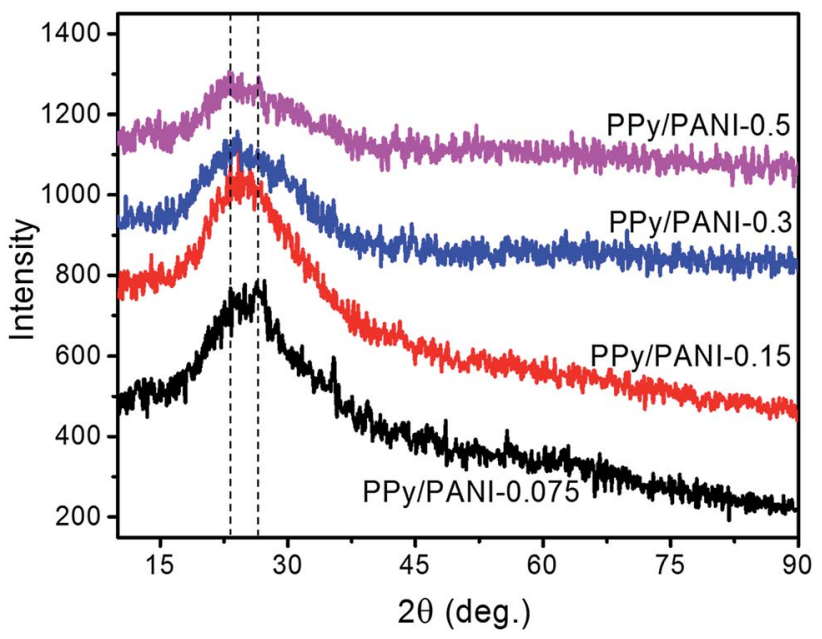

Fig. 3 XRD patterns of PPy/PANI composite films.
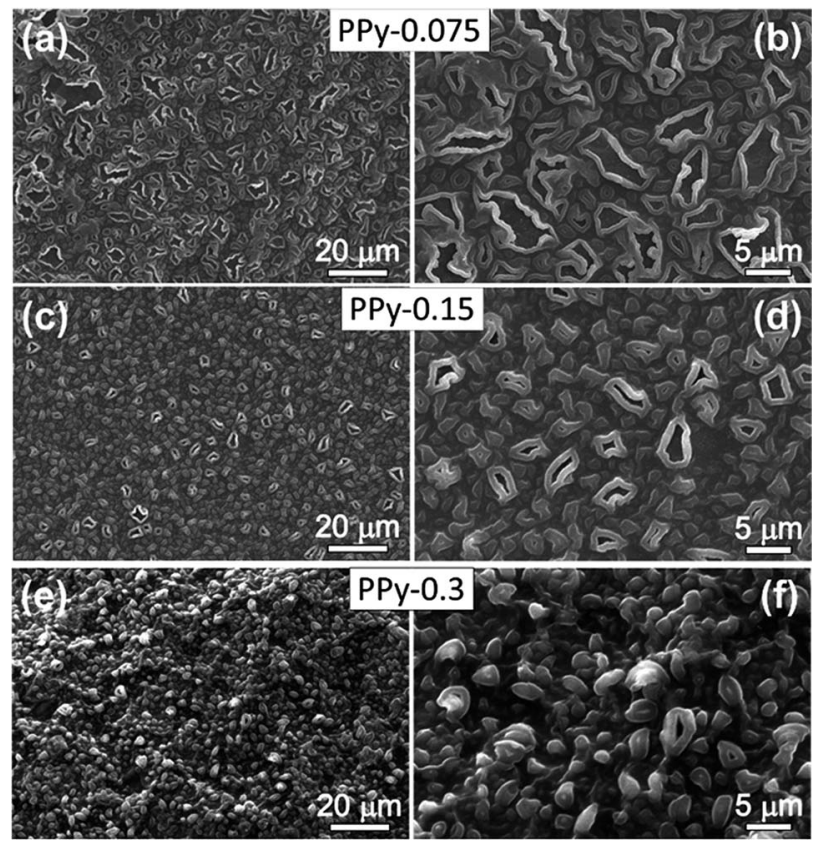

Fig. 4 SEM images of PPy films: (a and b) $0.075 \mathrm{M}$; (c and d) $0.15 \mathrm{M}$ and (e and f) $0.3 \mathrm{M}$.

nucleation rate of PPy. When the $\mathrm{FeCl}_{3}$ concentration was increased to $0.3 \mathrm{M}$ (Fig. $4 \mathrm{e}$ and $\mathrm{f}$ ), the edge of each feature tend to be knitted together to form particulate morphology.

Top-view and cross-sectional SEM images of PPy/PANI composite films prepared at different $\mathrm{FeCl}_{3}$ concentrations are shown in Fig. 5. PPy/PANI composite film prepared at the $\mathrm{FeCl}_{3}$ concentration of $0.075 \mathrm{M}$ represents various particles embedded on a membrane, which contains a large number of round-shaped pores with an average diameter of $c a .7 .5 \mu \mathrm{m}$ (Fig. 5a and b). Based on the morphology of PPy film (without PANI), it can be concluded that PANI particles have been distributed on PPy film support. At the $\mathrm{FeCl}_{3}$ concentration of $0.15 \mathrm{M}$ (Fig. $5 \mathrm{c}$ and d), the pore-on-membrane structure was not observed. On PPy film surface, there are particles with diameter smaller than $1 \mu \mathrm{m}$. When the $\mathrm{FeCl}_{3}$ concentration increases, the polymerization rate is also increased. As a result, PANI tends to form very small thin film stacked on PPy surface (Fig. 5e and f). It is thus concluded that at low concentration of $\mathrm{FeCl}_{3}$ catalyst, the composite film was formed due to the even distribution of PANI particles on PPy film, and at higher concentration of $\mathrm{FeCl}_{3}$ catalyst, the composite film is the combination of PPy film on PANI film. Fig. 5i reveals a typical cross-sectional SEM image of the PPy/PANI-0.5 film with a thickness of $c a .60 \mu \mathrm{m}$. Fig. 6 shows the typical EDX spectrum and the quantitative analysis of elemental composition of PPy/PANI-0.3 film. The main elements of interest including carbon, nitrogen, and oxygen were all detected. In addition, the presence of iron (Fe) and chlorine $(\mathrm{Cl})$ (despite our careful washing steps) indicates that a large number of $\mathrm{FeCl}_{3}$ oxidant molecules has been absorbed into the deep pores of the polymer backbones despite our thorough washing steps.

From the above analyses, the formation of PPy/PANI composite films can be explained as following: pyrrole/aniline 

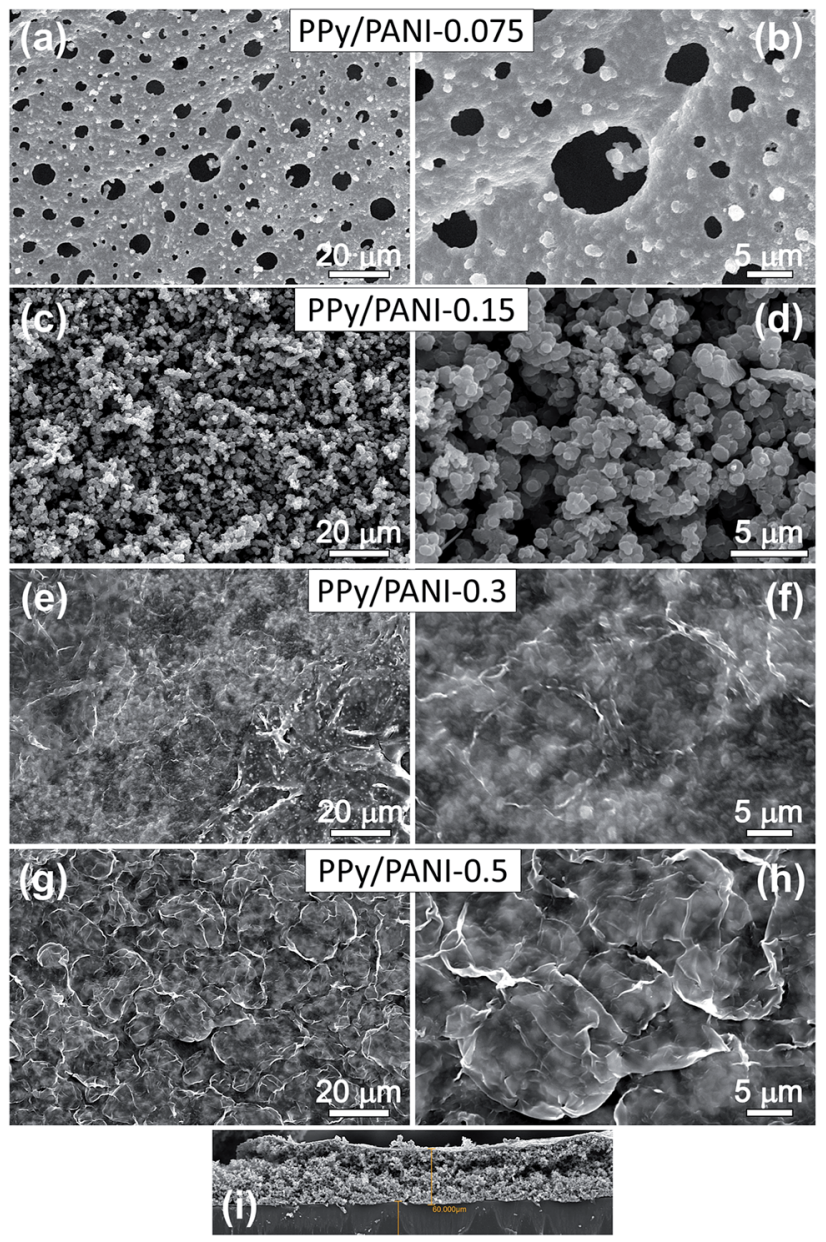

Fig. 5 Top-view SEM images of PPy/PANI composite films: ( $a$ and $b$ ) $0.075 \mathrm{M}$; (c and d) $0.15 \mathrm{M}$; (e and f) $0.3 \mathrm{M}$ and ( $\mathrm{g}$ and h) $0.5 \mathrm{M}$. Typical cross-sectional SEM image of PPy/PANI-0.5 composite films (i).

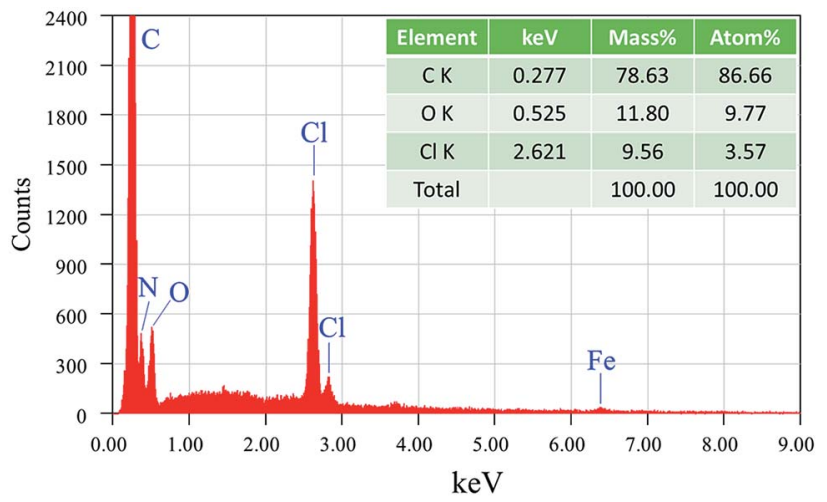

Fig. 6 EDX spectrum and quantitative analysis of elemental composition of PPy/PANI-0.3 composite film.

monomers and $\mathrm{FeCl}_{3}$ oxidant are brought into contact at the vapor/liquid interface and the polymerization occurs therein, firstly generating a thin polymer film on $\mathrm{FeCl}_{3}$ aqueous solution. After that, pyrrole and aniline monomers continually permeate through the pores of the as-formed polymer film and react with $\mathrm{FeCl}_{3}$ to form subsequent polymer layers. Due to its low density and hydrophobic surface, the PPy/PANI composite film is confined at the vapor/liquid interface as a floating film. The formation and evolution of microporous holes in the film could be attributed to the vacuum-induced evaporation of water through the growing film. The evaporation of water depends on $\mathrm{FeCl}_{3}$ concentration according to Raoult's second law of thermodynamics of molecular solution. When $\mathrm{FeCl}_{3}$ concentration increases, the saturated evaporation pressure of water decreases, then the porosity decreases. We note that the evaporation was principally induced by reduced pressure caused by using rotary pump.

On the other side, the morphology of the prepared PPy/PANI films might be closely associated with the partial pressure of each monomer in vapor phase. This aspect was considered by employing Antoine equation, which describes the relation between vapor pressure and temperature for pure components according to the formula:

$$
\log _{10} p=A-B /(T+C)
$$

where $p$ is the vapor pressure (in $\mathrm{mmHg}$ ), $T$ is the temperature (in Celsius), and $A, B$ and $C$ are component-specific constants. Using reported $A, B$, and $C$ data, ${ }^{39}$ the calculated vapor pressure of aniline and pyrrole at $25{ }^{\circ} \mathrm{C}$ are 0.465 and $8.288 \mathrm{mmHg}$, respectively. At this temperature, the vapor pressure of pyrrole is roughly 17.8 times higher than that of aniline. As a result, at the initial stage of the interfacial polymerization, a PPy film will be formed and acts as nucleation sites and support for the subsequent growth of PANI particles.

\subsection{The adsorption of $\operatorname{Cr}(\mathrm{vI})$}

The $\mathrm{pH}$ of initial solution might play an important role on $\mathrm{Cr}(\mathrm{vI})$ adsorption of PPy/PANI film, because pH determines which form of $\mathrm{Cr}(\mathrm{vI})$ exists in the solution. It has been reported that at $\mathrm{pH}$ 2.0-6.0, the predominant $\mathrm{Cr}(\mathrm{vI})$ species are monovalent bichromate $\left(\mathrm{HCrO}_{4}{ }^{-}\right)$and divalent dichromate $\left(\mathrm{Cr}_{2} \mathrm{O}_{7}{ }^{2-}\right)$, and at pH above $6.0, \mathrm{CrO}_{4}{ }^{2-}$ is the predominant species. ${ }^{40}$ We have investigated the influence of $\mathrm{pH}$ on the adsorption capacity of PPy/PANI composite films prepared at different $\mathrm{FeCl}_{3}$ concentrations. As shown in Fig. 7, all PPy/PANI composite films show relatively high removal efficiency of $c a .84-99 \%$ at different $\mathrm{pH}$

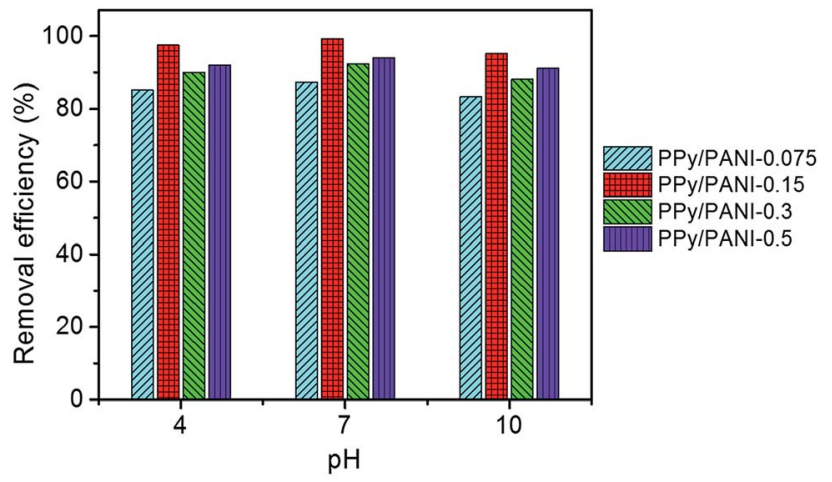

Fig. 7 Influence of $\mathrm{pH}$ on $\mathrm{Cr}(\mathrm{VI})$ adsorption capacity of PPy/PANI composites films. 
(4.0, 7.0, and 10.0). For each PPy/PANI composite films, the influence of $\mathrm{pH}$ on $\mathrm{Cr}(\mathrm{vI})$ removal efficiency is negligible, which means that there is no competitive interaction between hydroxyl $\left(\mathrm{OH}^{-}\right)$and $\mathrm{CrO}_{4}{ }^{2-}$ ions for the same sorption sites on the surface of the composite film. ${ }^{20}$ Our data suggests that these films could be used in a wide range of $\mathrm{pH}$.

Several adsorption isotherm models have been applied to analyze absorption data and evaluate $\mathrm{Cr}(\mathrm{vI})$ adsorption capacity of polymeric adsorbents. ${ }^{15,16,19,20}$ Among those, the most popular one is Langmuir isotherm, which assumes monolayer adsorption onto a surface containing a finite number of adsorption sites without transmigration of adsorbate in the plane of the surface. ${ }^{\mathbf{4 1}}$ The linearized Langmuir isotherm equation is represented as:

$$
C_{\mathrm{e}} / q_{\mathrm{e}}=C_{\mathrm{e}} / q_{\mathrm{m}}+1 / q_{\mathrm{m}} K_{\mathrm{L}}
$$

where $q_{\mathrm{e}}$ and $C_{\mathrm{e}}$ are the adsorption capacity $\left(\mathrm{mg} \mathrm{g}^{-1}\right)$ and concentration of $\mathrm{Cr}(\mathrm{vI})\left(\mathrm{mg} \mathrm{L}^{-1}\right)$ at equilibrium, respectively; $q_{\mathrm{m}}$ and $K_{\mathrm{L}}$ represent the maximum adsorption capacity of adsorbents $\left(\mathrm{mg} \mathrm{g}^{-1}\right)$ and the Langmuir constant $\left(\mathrm{L} \mathrm{mg}^{-1}\right)$, respectively. The values of $q_{\mathrm{m}}$ and $K_{\mathrm{L}}$ were calculated from the slope and intercept of the linear plot of $C_{\mathrm{e}} / q_{\mathrm{e}}$ versus $C_{\mathrm{e}}$.

The results obtained from adsorption isotherms for $\mathrm{Cr}(\mathrm{vI})$ adsorption onto PPy/PANI composite films are plotted in Fig. 8 and summarized in Table 1 . As shown in the table, the Langmuir correlation coefficients $\left(R^{2}\right)$ are very high $\left(R^{2}>0.99\right)$,

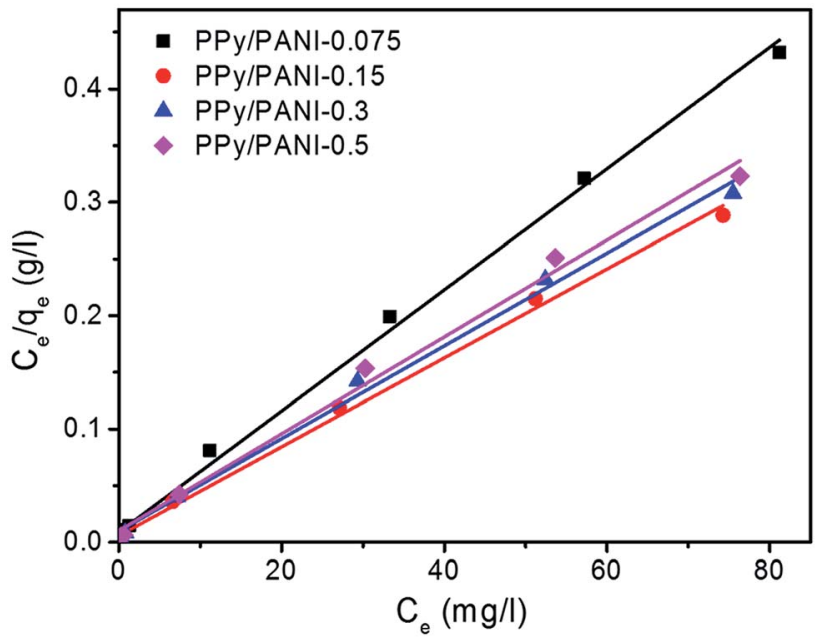

Fig. 8 Adsorption isotherm of $\mathrm{Cr}(\mathrm{VI})$ onto PPy/PANi (linearized Langmuir equations) at $T \sim 25^{\circ} \mathrm{C}, \mathrm{pH} 7.0$, PPy/PANI dosage $0.1 \mathrm{~g} \mathrm{~L}^{-1}$.

Table 1 The calculated parameters of the Langmuir isotherm model

\begin{tabular}{llll}
\hline & \multicolumn{3}{l}{ Langmuir isotherm } \\
\cline { 2 - 4 } PPy/PANI composite film & $q_{\mathrm{m}}\left(\mathrm{mg} \mathrm{g}^{-1}\right)$ & $K_{\mathrm{L}}\left(\mathrm{L} \mathrm{mg}^{-1}\right)$ & $R^{2}$ \\
\hline PPy/PANI-0.075 & 192.31 & 0.60 & 0.9935 \\
PPy/PANI-0.15 & 256.41 & 0.6747 & 0.9964 \\
PPy/PANI-0.3 & 243.90 & 0.4321 & 0.9951 \\
PPy/PANI-0.5 & 232.56 & 0.4207 & 0.9935
\end{tabular}

Table 2 Comparison on $q_{\max }$ values of different adsorbents

\begin{tabular}{llll}
\hline & $\begin{array}{l}q_{\max } \\
\left(\mathrm{mg} \mathrm{g}^{-1}\right)\end{array}$ & Optimal pH & Ref. \\
\hline Adsorbent & 15.47 & 4.0 & 42 \\
AC/poly(4-vinylpridine) & 53.7 & 2.25 & 43 \\
Diatomite-supported magnetite & 69.16 & 2.0 & 44 \\
nanoparticles & & & \\
Oxidized MWCNTs & 2.60 & 2.88 & 45 \\
PANI-PEG composite & 3.4 & 5.0 & 19 \\
PPy/Fe $\mathrm{O}_{4}$ nanocomposite & 169.4 & 2.0 & 46 \\
PPy-wood sawdust & 149.5 & $2.0 \div 6.0$ & 2 \\
Polyacrylonitrile/PPy nanofiber & 61.80 & 2.0 & 20 \\
PPy-PANI nanofibers & $227-294$ & $2.0 \div 8.0$ & 16 \\
PPy/PANI-0.15 composite film & 256.41 & 7 & This work
\end{tabular}

suggesting that the adsorption of $\mathrm{Cr}(\mathrm{vI})$ onto PPy/PANI composite films can be well described by monolayer adsorption model. The $q_{\mathrm{m}}$ values of all PPy/PANI composite films are all higher than $190 \mathrm{mg} \mathrm{g}^{-1}$, and PPy/PANI-0.15 film exhibits the highest $q_{\mathrm{m}}\left(256.41 \mathrm{mg} \mathrm{g}^{-1}\right)$. Table 2 shows $q_{\max }$ values of different adsorbents towards $\mathrm{Cr}(\mathrm{vI})$ adsorption, reported in the literature. Compared with other PPy-based materials, our PPy/ PANI composite films exhibited much higher $q_{\mathrm{m}}$, which is comparable to that of PPy-PANI nanofibers reported earlier. ${ }^{\mathbf{1 6}}$ Because of their excellent adsorption property, PPy/PANi composite films could be used as an effective adsorbent material for $\mathrm{Cr}(\mathrm{vI})$ removal from aqueous solution.

\section{Conclusions}

In summary, we have presented a simple and novel method for fabricating free-standing PPy/PANI composite films. The composite film is formed at the vapor/liquid interface by the interfacial polymerization reactions of gaseous pyrrole and aniline monomers in the presence of aqueous $\mathrm{FeCl}_{3}$ oxidant. The composite films have large density of PANI particles, covered with porous PPy membrane. The observed porosity is due to the evaporation and diffusion of water vapor through composite film, particularly at lower concentrations of $\mathrm{FeCl}_{3}$. The maximum $\mathrm{Cr}(\mathrm{vI})$ adsorption capacity of PPy/PANI composite film is $256.41 \mathrm{mg} \mathrm{g}^{-1}$, much higher than that of reported PPy-based adsorbents. Our interfacial polymerization method can be extended to fabricate other binary or ternary polymer composite films. Further works are being conducting to improve the mechanical performance of the PPy/PANI composite films while maintaining their advantageous properties. One promising approach is to incorporate PPy/PANI composite into mechanically strong supports such as carbon fibers.

\section{Conflicts of interest}

There are no conflicts of interest to declare. 


\section{Acknowledgements}

This research is funded by Vietnam National Foundation for Science and Technology Development (NAFOSTED) under grant number 103.02-2015.100. Mr Nguyen Thanh Vinh is acknowledged for his help with FTIR measurement.

\section{References}

1 D. Vu, Z. Li, H. Zhang, W. Wang, Z. Wang, X. Xu, B. Dong and C. Wang, Adsorption of $\mathrm{Cu}$ (II) from aqueous solution by anatase mesoporous $\mathrm{TiO}_{2}$ nanofibers prepared via electrospinning, J. Colloid Interface Sci., 2012, 367, 429-435.

2 R. Ansari and N. K. Fahim, Application of polypyrrole coated on wood sawdust for removal of $\mathrm{Cr}$ (VI) ion from aqueous solutions, React. Funct. Polym., 2007, 67, 367-374.

3 V. Gupta, S. Agarwal and T. A. Saleh, Chromium removal by combining the magnetic properties of iron oxide with adsorption properties of carbon nanotubes, Water Res., 2011, 45, 2207-2212.

4 V. Sarin and K. K. Pant, Removal of chromium from industrial waste by using eucalyptus bark, Bioresour. Technol., 2006, 97, 15-20.

5 K. Pillay, E. Cukrowska and N. Coville, Multi-walled carbon nanotubes as adsorbents for the removal of parts per billion levels of hexavalent chromium from aqueous solution, J. Hazard. Mater., 2009, 166, 1067-1075.

6 W. H. Organization, Guidelines for drinking-water quality: recommendations, World Health Organization, 2004.

7 C. E. Barrera-Díaz, V. Lugo-Lugo and B. Bilyeu, A review of chemical, electrochemical and biological methods for aqueous Cr (VI) reduction, J. Hazard. Mater., 2012, 223, 1-12.

8 K. Choi, S. Lee, J. O. Park, J.-A. Park, S.-H. Cho, S. Y. Lee, J. H. Lee and J.-W. Choi, Chromium removal from aqueous solution by a PEI-silica nanocomposite, Sci. Rep., 2018, 8, 1438.

9 S. Liu, X. Chen, X. Chen, Z. Liu and H. Wang, Activated carbon with excellent chromium (VI) adsorption performance prepared by acid-base surface modification, $J$. Hazard. Mater., 2007, 141, 315-319.

10 P. Wang and I. M. Lo, Synthesis of mesoporous magnetic $\gamma$ $\mathrm{Fe}_{2} \mathrm{O}_{3}$ and its application to $\mathrm{Cr}$ (VI) removal from contaminated water, Water Res., 2009, 43, 3727-3734.

11 Y. G. Ko, Y. J. Chun, C. H. Kim and U. S. Choi, Removal of Cu (II) and Cr (VI) ions from aqueous solution using chelating fiber packed column: equilibrium and kinetic studies, $J$. Hazard. Mater., 2011, 194, 92-99.

12 F. Gode and E. Pehlivan, Removal of Cr (VI) from aqueous solution by two Lewatit-anion exchange resins, J. Hazard. Mater., 2005, 119, 175-182.

13 D. Park, S.-R. Lim, Y.-S. Yun and J. M. Park, Development of a new $\mathrm{Cr}$ (VI)-biosorbent from agricultural biowaste, Bioresour. Technol., 2008, 99, 8810-8818.

14 A. N. Chowdhury, S. Jesmeen and M. Hossain, Removal of dyes from water by conducting polymeric adsorbent, Polym. Adv. Technol., 2004, 15, 633-638.
15 B. Qiu, C. Xu, D. Sun, Q. Wang, H. Gu, X. Zhang, B. L. Weeks, J. Hopper, T. C. Ho and Z. Guo, Polyaniline coating with various substrates for hexavalent chromium removal, Appl. Surf. Sci., 2015, 334, 7-14.

16 M. Bhaumik, A. Maity, V. Srinivasu and M. S. Onyango, Removal of hexavalent chromium from aqueous solution using polypyrrole-polyaniline nanofibers, Chem. Eng. J., 2012, 181, 323-333.

17 H. N. M. E. Mahmud, A. O. Huq and R. binti Yahya, The removal of heavy metal ions from wastewater/aqueous solution using polypyrrole-based adsorbents: a review, $R S C$ Adv., 2016, 6, 14778-14791.

18 M. Bhaumik, R. McCrindle and A. Maity, Efficient removal of Congo red from aqueous solutions by adsorption onto interconnected polypyrrole-polyaniline nanofibres, Chem. Eng. J., 2013, 228, 506-515.

19 M. R. Samani, S. M. Borghei, A. Olad and M. J. Chaichi, Removal of chromium from aqueous solution using polyaniline-poly ethylene glycol composite, J. Hazard. Mater., 2010, 184, 248-254.

20 J. Wang, K. Pan, Q. He and B. Cao, Polyacrylonitrile/ polypyrrole core/shell nanofiber mat for the removal of hexavalent chromium from aqueous solution, J. Hazard. Mater., 2013, 244, 121-129.

21 S. Virji, J. Huang, R. B. Kaner and B. H. Weiller, Polyaniline nanofiber gas sensors: examination of response mechanisms, Nano Lett., 2004, 4, 491-496.

22 H. Guan, L.-Z. Fan, H. Zhang and X. Qu, Polyaniline nanofibers obtained by interfacial polymerization for highrate supercapacitors, Electrochim. Acta, 2010, 56, 964-968.

23 P. Dallas and V. Georgakilas, Interfacial polymerization of conductive polymers: generation of polymeric nanostructures in a 2-D space, Adv. Colloid Interface Sci., 2015, 224, 46-61.

24 D. D. Sawall, R. M. Villahermosa, R. A. Lipeles and A. R. Hopkins, Interfacial polymerization of polyaniline nanofibers grafted to Au surfaces, Chem. Mater., 2004, 16, 1606-1608.

25 P. Dallas, D. Niarchos, D. Vrbanic, N. Boukos, S. Pejovnik, C. Trapalis and D. Petridis, Interfacial polymerization of pyrrole and in situ synthesis of polypyrrole/silver nanocomposites, Polymer, 2007, 48, 2007-2013.

26 C. Bora and S. Dolui, Fabrication of polypyrrole/graphene oxide nanocomposites by liquid/liquid interfacial polymerization and evaluation of their optical, electrical and electrochemical properties, Polymer, 2012, 53, 923-932.

27 J. Lei, Z. Li, X. Lu, W. Wang, X. Bian, T. Zheng, Y. Xue and C. Wang, Controllable fabrication of porous free-standing polypyrrole films via a gas phase polymerization, J. Colloid Interface Sci., 2011, 364, 555-560.

$28 \mathrm{H}$. Guo, H. Zhu, H. Lin and J. Zhang, Polyaniline/ $/ \mathrm{Fe}_{3} \mathrm{O}_{4}$ nanocomposites synthesized under the direction of cationic surfactant, Mater. Lett., 2008, 62, 2196-2199.

29 R. Rajagopalan and J. O. Iroh, Characterization of polyaniline-polypyrrole composite coatings on low carbon steel: a XPS and infrared spectroscopy study, Appl. Surf. Sci., 2003, 218, 58-69. 
$30 \mathrm{X} . \mathrm{Ou}$ and $\mathrm{X} . \mathrm{Xu}$, A simple method to fabricate poly (anilineco-pyrrole) with highly improved electrical conductivity via pre-polymerization, $R S C$ Adv. , 2016, 6, 13780-13785.

31 J. Stejskal, M. Trchová, I. A. Ananieva, J. Janča, J. Prokeš, S. Fedorova and I. Sapurina, Poly (aniline-co-pyrrole): powders, films, and colloids. Thermophoretic mobility of colloidal particles, Synth. Met., 2004, 146, 29-36.

32 P. Xu, X. Han, C. Wang, B. Zhang and H.-L. Wang, Morphology and physico-electrochemical properties of poly (aniline-co-pyrrole), Synth. Met., 2009, 159, 430-434.

33 C. Zhou, J. Han, G. Song and R. Guo, Fabrication of poly (aniline-co-pyrrole) hollow nanospheres with Triton X-100 micelles as templates, J. Polym. Sci., Part A: Polym. Chem., 2008, 46, 3563-3572.

34 P. Xu, X. Han, J. Jiang, X. Wang, X. Li and A. Wen, Synthesis and characterization of novel coralloid polyaniline/ BaFe12O19 nanocomposites, J. Phys. Chem. C, 2007, 111, 12603-12608.

35 B. Liang, Z. Qin, J. Zhao, Y. Zhang, Z. Zhou and Y. Lu, Controlled synthesis, core-shell structures and electrochemical properties of polyaniline/polypyrrole composite nanofibers, J. Mater. Chem. A, 2014, 2, 2129-2135.

36 M. Ortuño, E. Escasain, E. Lopez-Elvira, A. M. Somoza, J. Colchero and E. Palacios-Lidon, Conducting polymers as electron glasses: surface charge domains and slow relaxation, Sci. Rep., 2016, 6, 21647.

37 G. Qi, Z. Wu and H. Wang, Highly conductive and semitransparent free-standing polypyrrole films prepared by chemical interfacial polymerization, J. Mater. Chem. C, 2013, 1, 7102-7110.
38 J. Liu and M. Wan, Synthesis, characterization and electrical properties of microtubules of polypyrrole synthesized by a template-free method, J. Mater. Chem., 2001, 11, 404-407.

39 C. L. Yaws, The Yaws Handbook of Vapor Pressure: Antoine Coefficients, Gulf Professional Publishing, 2015.

$40 \mathrm{~J}$. Hu, G. Chen and I. M. Lo, Removal and recovery of Cr (VI) from wastewater by maghemite nanoparticles, Water Res., 2005, 39, 4528-4536.

41 K. Y. Foo and B. H. Hameed, Insights into the modeling of adsorption isotherm systems, Chem. Eng. J., 2010, 156, 2-10.

$42 \mathrm{~S}$. Babel and T. A. Kurniawan, Cr (VI) removal from synthetic wastewater using coconut shell charcoal and commercial activated carbon modified with oxidizing agents and/or chitosan, Chemosphere, 2004, 54, 951-967.

43 J. Fang, Z. Gu, D. Gang, C. Liu, E. S. Ilton and B. Deng, Cr (VI) removal from aqueous solution by activated carbon coated with quaternized poly (4-vinylpyridine), Environ. Sci. Technol., 2007, 41, 4748-4753.

44 P. Yuan, D. Liu, M. Fan, D. Yang, R. Zhu, F. Ge, J. Zhu and H. He, Removal of hexavalent chromium [Cr (VI)] from aqueous solutions by the diatomite-supported/ unsupported magnetite nanoparticles, J. Hazard. Mater., 2010, 173, 614-621.

45 J. Hu, C. Chen, X. Zhu and X. Wang, Removal of chromium from aqueous solution by using oxidized multiwalled carbon nanotubes, J. Hazard. Mater., 2009, 162, 1542-1550.

46 M. Bhaumik, A. Maity, V. Srinivasu and M. S. Onyango, Enhanced removal of $\mathrm{Cr}$ (VI) from aqueous solution using polypyrrole $/ \mathrm{Fe}_{3} \mathrm{O}_{4}$ magnetic nanocomposite, J. Hazard. Mater., 2011, 190, 381-390. 